\title{
PALLADIUM(0) CATALYZED ISOMERIZATION OF ALKYNYLDIETHYL PHOSPHONATE AND ALKYNYLDIPHENYL PHOSPHINE OXIDE
}

\author{
Chunlin Ma*1, Xiyan Lu² and Yongxiang $\mathrm{Ma}^{3}$ \\ 1 Department of Chemistry, Liaocheng Teachers College, \\ Liaocheng 252059, People's Republic of China \\ 2 Shanghai Institute of Organic Chemistry, Academia Sinica \\ 3 Department of Chemistry, Lanzhou University, Lanzhou 730000, People's Republic of China
}

\section{Introduction}

The isomerization of alkynes to conjugated dienes can represent a useful synthetic approach to these valuable blocks due to the ready accessibility and elaboration of alkynes. Recently there have appeared seveal reports about the isomerizations of alkynones[1], alkynoic esters[2], alkynoic amides [3] and propargylic alcohols [4] catalyzed by transition metal complexes. We have found that an $\alpha$-electronwithdrawing carbonyl group is important for this reaction. In order to examine the involvement of an aelectron-withdrawing group, we decided to study the isomerization of acetylenic derivatives with other electron withdrawing group, such as the $P(O)(O R)_{2}, P(O) P_{2}$ groups. We wish to report here the isomerizations of alkynyldiethyl phosphonate and alkynyldiphenyl phosphine oxide catalyzed by palladium.

2. Results and discussion

2. 1. The results of the isomerization were shown in Scheme 1 .

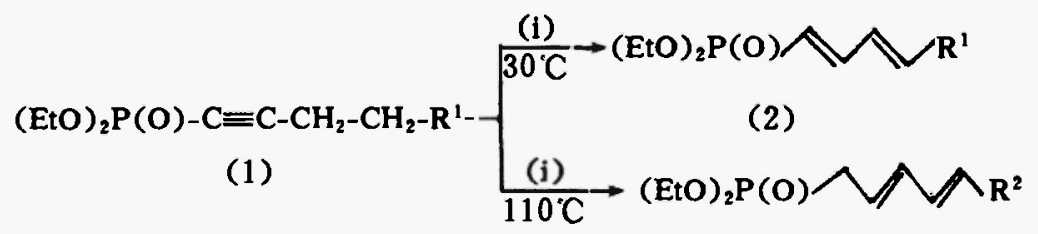

(3)

$$
\begin{array}{rccc}
1 a: R^{1}=C_{3} ; & 1 b: R^{1}=C_{2} H_{5} ; & 1 c: R^{1}=n-C_{3} H_{7} ; & 1 d ; R^{1}=n-C_{4} H_{9} . \\
2 a: R^{1}=C_{3} ; & 2 b: R^{1}=C_{2} H_{5} ; & 2 c: R^{1}=n-C_{3} H_{7} ; & 2 d: R^{1}=n-C_{4} H_{8} . \\
& 3 b: R^{2}=C_{3} ; & 3 c: R^{2}=C_{2} H_{5}, & 3 d: R^{2}=n-C_{3} H_{7} .
\end{array}
$$$$
\mathrm{Ph}_{2} \mathrm{P}(\mathrm{O}) \mathrm{C}=\mathrm{C}-\mathrm{CH}_{2}-\mathrm{CH}_{2}-\mathrm{R}^{1} \frac{\text { (i) }}{30 \mathrm{C} \text { or } 110 \mathrm{C}} \longrightarrow \mathrm{Ph}_{2} \mathrm{P}(\mathrm{O}) \longrightarrow \mathrm{R}^{1}
$$

$4 a: R^{1}=C_{3} ; 4 b: R^{1}=C_{2} H_{5} ; 4 c: R^{1}=n-C_{3} H_{7} ; 4 d: R^{1}=n-C_{1} H_{8}$.

$5 a ! R^{1}=C_{3} ; 5 b: R^{1}=C_{2} H_{5} ; 5 c: R^{\prime}=n-C_{3} H_{7} ; 5 d: R^{1}=n-C_{4} H_{9}$. Scheme 1.

(i) $1 \mathrm{mmol}$ (1) or (4), $0.05 \mathrm{mmol} \mathrm{Pd}_{2}$ (dba), $\cdot \mathrm{CH}_{3} \mathrm{Cl}, 0.20 \mathrm{mmol} \mathrm{i-Pr}{ }_{3} \mathrm{P}$, $1 \mathrm{mmol}$ HOAc, toluene. dba $=$ dibenylideneacetone. 


\section{2. Effect of reaction temperature}

The results of these thermal isomerizations are given in Table 1. The thermal isomerization of alkynyldjethyl phosphonates $1 \mathrm{a}$ to $1 \mathrm{~d}$ occured at $30^{\circ} \mathrm{C}$ to yjeld the $1 \mathrm{E}, 3 \mathrm{E}$-alkadienyl (diethyl) phosphonates $2 \mathrm{a}$ to $2 \mathrm{~d}$; whereas, at $110^{\circ} \mathrm{C}$, isomeric $2 \mathrm{E}, 4 \mathrm{E}$-alkadjenyl (diethyl) phosphonates $3 \mathrm{~b}$ to $3 \mathrm{~d}$ are formed. Compound la is transformed into $2 \mathrm{a}$ only, even at $110^{\circ} \mathrm{C}$, which can be explained by the lower stabjlity of a terminal double bond that would arise if $2 \mathrm{a}$ would isomerize, as $2 \mathrm{~b}, 2 \mathrm{c}$, or $2 \mathrm{~d}$ do. Analogously, the isomerization of alkynyldiphenyl phosphine oxides $4 \mathrm{a}$ to $4 \mathrm{~b}$ yjelded only the $1 \mathrm{E}, 3 \mathrm{E}$-alkadjenyl (diphenyl) phosphine oxides 5 a to $5 \mathrm{~d}$, which might be due the donor ability of the phenyl moiety in compounds of type 4 .

TABLE 1. Isomerization of (1) and (4) effected by reaction temperature

\begin{tabular}{|c|c|c|c|c|}
\hline Compounds & $\begin{array}{c}\text { Reaction } \\
\text { Temp. ( C ) }\end{array}$ & $\begin{array}{l}\text { Reaction } \\
\text { Time(h) }\end{array}$ & Product & $\begin{array}{c}\text { Isolated yield } \\
(\%)\end{array}$ \\
\hline la & 30 & 58 & $2 a$ & 85 \\
\hline lb & 30 & 64 & $2 b$ & 86 \\
\hline lc & 30 & 67 & $2 c$ & 79 \\
\hline Id & 30 & 69 & $2 d$ & 82 \\
\hline la & 110 & 24 & $2 a$ & 89 \\
\hline $\mathrm{lb}$ & 110 & 30 & $3 b$ & 92 \\
\hline $1 c$ & 110 & 27 & $3 c$ & 89 \\
\hline 1d & 110 & 34 & $3 d$ & 90 \\
\hline $4 a$ & 30 & 64 & $5 a$ & 83 \\
\hline $4 a$ & 110 & 25 & $5 a$ & 87 \\
\hline $4 b$ & 30 & 72 & $5 b$ & 87 \\
\hline $4 b$ & 110 & 30 & $5 b$ & 86 \\
\hline $4 c$ & 30 & 70 & $5 c$ & 85 \\
\hline $4 c$ & 110 & 30 & $5 c$ & 87 \\
\hline $4 d$ & 30 & 68 & $5 d$ & 84 \\
\hline $4 d$ & 110 & 28 & $5 d$ & 89 \\
\hline
\end{tabular}

\section{3. Stereochemistry of the isomerization}

The stereochemistry of the products can be determined by ${ }^{1} \mathrm{H}$ NMR and IR spectra. The diene geometry of compounds (2a-2d) was all E, E-form, due to their ${ }^{1} \mathrm{H}$ NMR and IR spectra were as same as in the literature[5]. For (3b) (EtO) ${ }_{2} \mathrm{P}(\mathrm{O})-\mathrm{CH}_{2}-\mathrm{CH}_{\mathrm{a}}=\mathrm{CH}_{\mathrm{b}}-\mathrm{CH}_{\mathrm{c}}=\mathrm{CH}_{\mathrm{a}}-\mathrm{CH}_{3}$, the coupling constants $J_{a, b}$ and $J_{c, d}$ were both about $15 \mathrm{~Hz}$; For $(5 a-5 d) P h, P(O)-C_{r}=C H_{z}-C H_{1}=C H_{j}-R^{1}$, the $J_{i, z}$ and $J_{1, j}$ were all about $14 \mathrm{~Hz}$; in addition, the IR spectra of (3b-3d) and (5a-5d) showed a characteristic band about $960,985 \mathrm{~cm}^{-1}$ respectively, due to the trans-trans alkadienyl moiety. We can conclude from this that the diene of (3b-3d) or (5a-5d) are E, E-type, which shows the high stereospecifity of the jsomerization, as shown previously $[1 \mathrm{c}, \mathrm{Id}]$. 


\section{4. Reaction mechanism}

The mechanism proposed for the isomerization of the substrate (1) at $30^{\circ} \mathrm{C}$ and (4) at $30^{\circ} \mathrm{C}$ or $110 \mathrm{C}$ were similar to that we have explained for the reaction of $\alpha, \beta-y$-yones[1c,6], it was an addition and elimination reaction of the $[\mathrm{PdH}]$ active specis, as outline in Scheme 2.

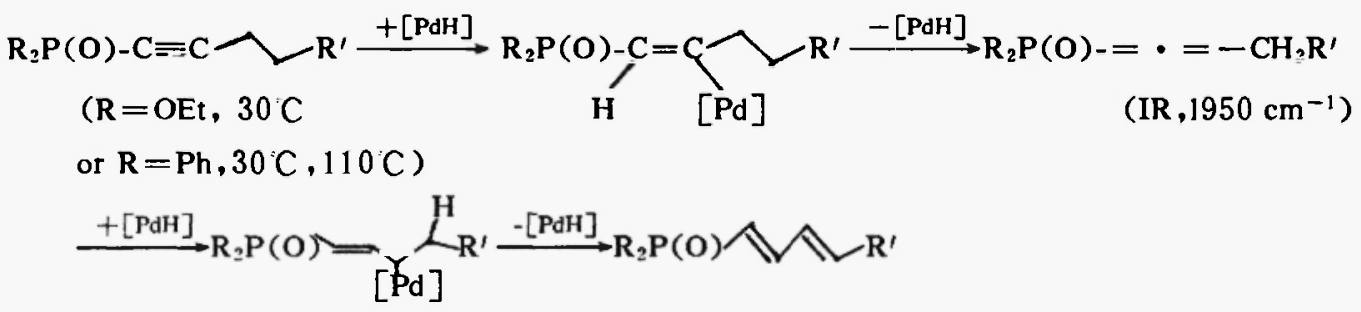

Scheme 2.

$[\mathrm{PdH}]=\mathrm{LmPdH}$, it represents the active specise containing a Pd-H bond. The $\mathrm{L}$ represents $\mathrm{dba}$, $\mathrm{i}-\mathrm{Pr}_{3} \mathrm{P}$ or solvent.

According to B. N. Noknh 1,2- butadienyldiethyl phosphonate exists as an equilibrium mixture of three isomers at $100 \mathrm{C}[7]$ :

$$
\begin{array}{ccc}
\mathrm{CH}_{3} \mathrm{CH}=\mathrm{C}=\mathrm{CH}-\mathrm{P}(\mathrm{O})(\mathrm{OEt})_{2} \rightleftharpoons \mathrm{CH}_{3} \mathrm{C} \equiv \mathrm{C}_{2} \mathrm{CH}_{2} \mathrm{P}(\mathrm{O})(\mathrm{OEt})_{2} \rightleftharpoons \mathrm{CH}_{3} \mathrm{CH}_{2} \mathrm{C} \equiv \mathrm{C}-\mathrm{P}(\mathrm{O})(\mathrm{OEt})_{2} \\
20 \% & 70 \% & 10 \%
\end{array}
$$

The fact that the 2-butynyldiethyl phosphonate is the main one can be due to conjugative ability of 2-butynyl with phosphorus was more favorable than 1,2-butadienyl or 1-butynyl[7]. Then the reaction mechanism of substrate (1) at $110^{\circ} \mathrm{C}$ can be explained in Scheme 3.

$$
(\mathrm{EtO})_{2}(\mathrm{O}) \mathrm{P}-\mathrm{C}=\mathrm{C}-\mathrm{CH}_{2}-\mathrm{CH}_{2}-\mathrm{R}^{1} \frac{+[\mathrm{PdH}]}{-[\mathrm{PdH}]}(\mathrm{EtO}),(\mathrm{O}) \mathrm{P}-=\cdot=\mathrm{CH}_{2}-\mathrm{R}^{1}
$$

$$
\stackrel{110 \mathrm{C}}{=}(\mathrm{EtO})_{2}(\mathrm{O}) \mathrm{P}-\mathrm{CH}_{2}-\mathrm{C}=\mathrm{C}-\mathrm{CH}, \mathrm{R}^{1} \frac{+[\mathrm{PdH}]}{-[\mathrm{PdH}]}(\mathrm{EtO})_{2}(\mathrm{O}) \mathrm{P}>\mathrm{R}^{2}
$$

$(3 b-3 d)$

Scheme 3

The substrate (1) first occured addition reaction with [ $\mathrm{PdH}]$, then lost it to form intermediate (6) The (6) was isomerized to $(7)$ at $110^{\circ} \mathrm{C}$, the (7) continued to occur isomerization catalyzed by palladium to give (3b-3d). In order to define the intermediate ( 7 ), we prepared the 2 -hexynyldiethyl phosphonate ( 8 ), which can be isomerized to form $2 \mathrm{E}, 4 \mathrm{E}$-hexadienyldiethyl phosphonate (3b) catalyzed by the palladium complex at $110^{\circ} \mathrm{C}$. as shown in Scheme 4 .

$$
\mathrm{CH}_{3} \mathrm{CH}_{2} \mathrm{CH}_{2} \mathrm{C} \equiv \mathrm{CCH}_{2} \mathrm{Br}+(\mathrm{EtO})_{2} \mathrm{P}(\mathrm{O}) \mathrm{H} \stackrel{\text { (ii) }}{\longrightarrow}(\mathrm{EtO})_{2}(\mathrm{O}) \mathrm{PCH}_{2} \mathrm{C} \equiv \mathrm{CCH}_{2} \mathrm{CH}_{2} \mathrm{CH}_{3}
$$

$$
\underset{110^{\circ} \mathrm{C}}{\stackrel{(i)}{\longrightarrow}}(\mathrm{EtO})_{2}(\mathrm{O}) \mathrm{P} \sim \mathrm{CH}_{3}
$$

Scheme 4. (ii) $\mathrm{THF}, \mathrm{NaN}\left(\mathrm{SiMe}_{3}\right)_{2},-10^{\circ} \mathrm{C}$ 
To summarize : alkynyldiethyl phosphonates or alkynyldiphenyl phosphine oxides isomerize in high yield and highly stereoselectively. The $2 \mathrm{E}, 4 \mathrm{E}$-alkadienyl (diethyl) phosphonates $3 \mathrm{~b}, 3 \mathrm{c}$, and $3 \mathrm{~d}$ can be used as synthons in organic synthesis[8].

\section{Experimental section}

All reactions were carried out under a prepurified argon atmosphere. Toluene was distilled from sodjum and benzophenone under nitrogen atomsphere. 'H-NMR were recorded on a Varian XL-200 or Bruker MSL-300 spectrometer. Chemical shifts were reported as values in parts per million with $\mathrm{Me}_{4} \mathrm{Si}$ as an internal standard. ${ }^{31} \mathrm{P}$ - NMR spectra were recorded on a JEOL-FX-90Q FT NMR spectrometer and referenced to $\mathrm{H}_{3} \mathrm{PO}_{4}(85 \%)$ contained in a coaxial tube and measured under conditions of complete ${ }^{1} \mathrm{H}$ decoupling. Infrared spectra were taken as liquid film or KBr disc with Shimadzu IR-440 instrument. Mass spectral data were obtained with electron ionization on a Finnigan 4021 spectrometer.

\subsection{Material:}

Alkynyldiethyl phosphonate (1) and alkynyldiphenyl phosphine oxide (4) were prepared according the reported methods[9], all their 'H- NMR, IR and MS spectra were determined.

Preparation of 2-hexynyldjethyl phosphonate ( 8 ) referenced to literature[10]. Oil liquid, yield $94 \%$. IR (neat) : $2900,2200,1390,1250,1020 \mathrm{~cm}^{-1}$. ${ }^{1} \mathrm{H}-\mathrm{NMR}\left(\mathrm{CDCl}_{3} / 200 \mathrm{MHz}\right) \cdot 4.2(\mathrm{~m}, 4 \mathrm{H})$, $2.70(\mathrm{~d}, \mathrm{~J} 21 \mathrm{~Hz}, 2 \mathrm{H}), 2.2(\mathrm{~m}, 2 \mathrm{H}), 1.5(\mathrm{~m}, 2 \mathrm{H}), 1.3(\mathrm{t}, \mathrm{J} 6 \mathrm{~Hz}, 6 \mathrm{H}), 1.0(\mathrm{t}, \mathrm{J} 7 \mathrm{~Hz}, 3 \mathrm{H}) \mathrm{ppm}$. MS: $\mathrm{m} / \mathrm{e} 218\left(\mathrm{M}^{+}, 100\right), 190(90), 134(50), 109(62), 81(70), 43(38)$. Anal. Calcd for $\mathrm{C}_{10} \mathrm{H}_{18} \mathrm{O}_{3} \mathrm{P}$ : C, 55.04; H, 8. 78; Found. C, 55.16; H, 8.92.

3. 2. General procedure for the isomerization of (1), (4) and (8) catalyzed by palladium complex $\mathrm{Pd}_{2}(\mathrm{dba})_{3} \mathrm{CHCl}_{3}$.

Under argon, a mixture of (1), (4) or (8) (lmmol), $\mathrm{HOAc}(1 \mathrm{mmol}), \mathrm{i}-\mathrm{Pr}_{3} \mathrm{P}(0.2 \mathrm{mmol}), \mathrm{Pd}_{2}$ (dba) ${ }_{3} \mathrm{CHCl}_{3}(0.05 \mathrm{mmol})$ and toluene $(5 \mathrm{ml})$ was heated at $30^{\circ} \mathrm{C}$ or $110^{\circ} \mathrm{C}$, as monitored by TLC. After cooling and removal of solvent, the residue chromatographed on a silica gel column ( eluting with acetone and petroleum ether) to give (2), (3), (5).

3. 2. 1. $1 \mathrm{E}, 3 \mathrm{E}$-pentadienyldiethyl phosphonate (2a) [10]

Pale yellow oil. IR (neat) : $2950,1640,1590,1250,1030,970 \mathrm{~cm}^{-1} .{ }^{1} \mathrm{H}-\mathrm{NMR}\left(\mathrm{CDCl}_{3} / 200\right.$ $\mathrm{MHz}$ ) : 7.07 (ddd, J $10,17,21 \mathrm{~Hz}, 1 \mathrm{H}), 6.10(\mathrm{~m}, 2 \mathrm{H}), 5.60$ (dd, J $17,19.5 \mathrm{~Hz}, 1 \mathrm{H}), 4.10(\mathrm{~m}$, $4 \mathrm{H})$, $1.85(\mathrm{~d}, \mathrm{~J} 5.4 \mathrm{~Hz}, 3 \mathrm{H}), 1.20(\mathrm{t}, \mathrm{J} 7 \mathrm{~Hz}, 6 \mathrm{H}) \mathrm{ppm} .{ }^{31} \mathrm{P}-\mathrm{NMR}\left(\mathrm{CDCl}_{3} / 90 \mathrm{MHz}\right): 19.85$ ppm. MS. m/e 204( $\left.\mathrm{M}^{+}, 19\right), 163(61), 135(100), 81(59), 43(71)$.

\section{2. 2. 1E, 3E-hexadienyldiethyl phosphonate (2b) [11]}

Pale yellow oil. IR (neat): $3000,1645,1600,1250,1040,975 \mathrm{~cm}^{-1} .{ }^{1} \mathrm{H}-\mathrm{NMR}\left(\mathrm{CDCl}_{3} / 200\right.$ $\mathrm{MHz}$ ) 7.10 (ddd, J 10, 16.8, $20 \mathrm{~Hz}, 1 \mathrm{H}), 6.12(\mathrm{~m}, 2 \mathrm{H}), 5.56(\mathrm{dd}, \mathrm{J} 17,20 \mathrm{~Hz}, \mathrm{HH}), 4.10(\mathrm{~m}$, $4 \mathrm{H}), 2.20(\mathrm{~m}, 2 \mathrm{H}), 1.30(\mathrm{t}, \mathrm{J} 7 \mathrm{~Hz}, 6 \mathrm{H}), 1.0(\mathrm{t}, \mathrm{J} 7 \mathrm{~Hz}, 3 \mathrm{H}) \mathrm{ppm} .{ }^{31} \mathrm{P}-\mathrm{NMR}\left(\mathrm{CDCl}_{3} / 90 \mathrm{MHz}\right)$ : 19. 94 ppm. MS: m/e $218\left(\mathrm{M}^{+}, 64\right), 189(43), 176(45), 148(100), 147(77), 120(47), 79$ 
(46).

3. 2. 3. 1E, 3E-heptadienyldiethyl phosphonate (2c)

Pale yellow oil. IR (neat) : $2930,1620,1570,1210,1020,965 \mathrm{~cm}^{-1} . \quad{ }^{1} \mathrm{H}-\mathrm{NMR}\left(\mathrm{CDCl}_{3} / 200\right.$ $\mathrm{MHz}$ ) 7.08 (ddd, J 10, 17, $21 \mathrm{~Hz}, 1 \mathrm{H}), 6.15(\mathrm{~m}, 2 \mathrm{H}), 5.60$ (dd, J $17,20 \mathrm{~Hz}, 1 \mathrm{H}), 4.10(\mathrm{~m}$, $4 \mathrm{H}), 2.10(\mathrm{~m}, 2 \mathrm{H}), 1.35(\mathrm{~m}, 3 \mathrm{H}), 0.95(\mathrm{t}, \mathrm{J} 7 \mathrm{~Hz}, 3 \mathrm{H}) \mathrm{ppm} .{ }^{31} \mathrm{P}-\mathrm{NMR}\left(\mathrm{CDCl}_{3} / 90 \mathrm{MHz}\right): 20.0$ ppm. MS: $\mathrm{m} / \mathrm{e} 233\left(\mathrm{M}^{+}+1,100\right), 232\left(\mathrm{M}^{+}, 12\right), 189(30), 177(27), 79(24)$. Anal. Calcd for $\mathrm{C}_{11}$ $\mathrm{H}_{21} \mathrm{O}_{3} \mathrm{P}: \mathrm{C}, 56.89 ; \mathrm{H}, 9.11$; Found: $\mathrm{C}, 56.93 ; \mathrm{H}, 8.98$.

\section{2. 4. 1E, 3E-octadienyldiethyl Phosphonate (2d)}

Pale yellow oil. IR (neat): $2950,1640,1600,1250,1040,960 \mathrm{~cm}^{-1}$. ${ }^{1} \mathrm{H}-\mathrm{NMR}\left(\mathrm{C}_{6} \mathrm{D}_{6} / 200\right.$ $\mathrm{MHz}): 7.30$ (ddd, J $10,17,21 \mathrm{~Hz}, 1 \mathrm{H}), 5.80(\mathrm{dd}, \mathrm{J} 17,20 \mathrm{~Hz}, 1 \mathrm{H}), 5.6(\mathrm{~m}, 2 \mathrm{H}), 4.0(\mathrm{~m}$, $4 \mathrm{H}), 1.5(\mathrm{~m}, 2 \mathrm{H}), 1.05(\mathrm{~m}, 13 \mathrm{H}) \mathrm{ppm}$. ${ }^{31} \mathrm{P}-\mathrm{NMR}\left(\mathrm{CDCl}_{3} / 90 \mathrm{MHz}\right): 19.83 \mathrm{ppm}$. MS : m/e 246 $\left(\mathrm{M}^{+}, 29\right), 189(100), 176(54), 120(64), 65(55)$. Anal. Caled for $\mathrm{C}_{12} \mathrm{H}_{23} \mathrm{O}_{3} \mathrm{P}: \mathrm{C}, 58.52 ; \mathrm{H}, 9$. 41; Found: C, 58. 97; H, 9. 62 .

\section{2. 5. $2 \mathrm{E}, 4 \mathrm{E}$-hexadienyldiethyl phosphonate (3b) [12]}

Colorless liquid. IR (neat): $2950,1640,1595,1250,1050,965 \mathrm{~cm}^{-1}$. ${ }^{1} \mathrm{H}-\mathrm{NMR}\left(\mathrm{CDCl}_{3} / 300\right.$ $\mathrm{MHz}): 6.20(\mathrm{~m}, 1 \mathrm{H}), 6.05$ (ddd, J $2.5,7,15 \mathrm{~Hz}, 1 \mathrm{H}), 5.70(\mathrm{~m}, 1 \mathrm{H}), 5.50(\mathrm{dd}, \mathrm{J} 7,14.5$ $\mathrm{Hz}, 1 \mathrm{H}), 4.10(\mathrm{~m}, 4 \mathrm{H}), 2.70(\mathrm{dd}, \mathrm{J} 8,23 \mathrm{~Hz}, 2 \mathrm{H}), 1.80(\mathrm{~d}, \mathrm{~J} 7 \mathrm{~Hz}, 3 \mathrm{H}), 1.30(\mathrm{t}, \mathrm{J} 7.5 \mathrm{~Hz}, 6 \mathrm{H})$ ppm. ${ }^{31} \mathrm{P}-\mathrm{NMR}\left(\mathrm{CDCl}_{3} / 90 \mathrm{MHz}\right): 27.39 \mathrm{ppm}$. MS: $\mathrm{m} / \mathrm{e} 218\left(\mathrm{M}^{+}, 100\right), 190(18), 162(21), 109$ (24), 80(79).

\section{2. 6. $2 \mathrm{E}, 4 \mathrm{E}$-heptadienyldiethyl phosphonate (3c)}

Colorless oil. IR (neat): $2950,1640,1595,1250,1030,960 \mathrm{~cm}^{-1} .{ }^{1} \mathrm{H}-\mathrm{NMR}\left(\mathrm{CDCl}_{3} / 200\right.$ $\mathrm{MHz}): 6.10(\mathrm{~m}, 2 \mathrm{H}), 5.60(\mathrm{~m}, 2 \mathrm{H}), 4.10(\mathrm{~m}, 4 \mathrm{H}), 2.65(\mathrm{dd}, \mathrm{J} 7.5,24 \mathrm{~Hz}, 2 \mathrm{H}), 1.8(\mathrm{~m}, 2 \mathrm{H})$, 1. $25(\mathrm{t}, \mathrm{J} 7 \mathrm{~Hz}, 6 \mathrm{H}), 0.95(\mathrm{t}, \mathrm{J}$ 7. $5 \mathrm{~Hz}, 3 \mathrm{H}) \mathrm{ppm} .{ }^{31} \mathrm{P}-\mathrm{NMR}\left(\mathrm{CDCl}_{3} / 90 \mathrm{MHz}\right): 27.48 \mathrm{ppm}$.

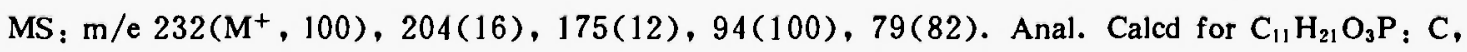
$56.89 ; \mathrm{H}, 9.11$; Found; C, 56. 48; H, 9. 34 .

\section{2. 7. $2 \mathrm{E}, 4 \mathrm{E}$-octadienyldiethyl phosphonate (3d) [12]}

Colorless oil. IR (neat) $2951,1642,1595,1249,1040,960 \mathrm{~cm}^{-1} .{ }^{1} \mathrm{H}-\mathrm{NMR}\left(\mathrm{CDCl}_{3} / 200\right.$ $\mathrm{MHz}): 6.10(\mathrm{~m}, 2 \mathrm{H}), 5.40(\mathrm{~m}, 2 \mathrm{H}), 4.0(\mathrm{~m}, 4 \mathrm{H}), 2.60(\mathrm{dd}, \mathrm{J} 8,22 \mathrm{~Hz}, 2 \mathrm{H}), 1.80(\mathrm{~m}, 2 \mathrm{H})$, 1. $20(\mathrm{~m}, 8 \mathrm{H}), 0.95(\mathrm{t}, \mathrm{J} 7.5 \mathrm{~Hz}, 3 \mathrm{H}) \mathrm{ppm}$. ${ }^{31} \mathrm{P}-\mathrm{NMR}\left(\mathrm{CDCl}_{3} / 90 \mathrm{MHz}\right): 27.41 \mathrm{ppm} . \mathrm{MS}: \mathrm{m} / \mathrm{e}$ $246\left(\mathrm{M}^{+}, 100\right), 217(30), 189(21), 108(57), 79(98), 59(52)$.

\section{2. 8. 1E, 3E-pentadienyldiphenyl phosphine oxide (5a) [13]}

White crystal, m. p. $155-156^{\circ} \mathrm{C}$ (1iterature [13] - m. p. $\left.154-155^{\circ} \mathrm{C}\right) . \mathrm{IR}(\mathrm{KBr}): 2950,1645$, $1590,1170,1120,985 \mathrm{~cm}^{1}$. ${ }^{1} \mathrm{H}-\mathrm{NMR}\left\{\left(\mathrm{CD}_{3}\right)_{2} \mathrm{CO} / 200 \mathrm{MHz}\right\} 7.75(\mathrm{~m}, 4 \mathrm{H}), 7.5(\mathrm{~s}, 6 \mathrm{H}), 7.1$ (ddd, J $10,17,18.5 \mathrm{~Hz}, 1 \mathrm{H}), 6.45(\mathrm{~m}, 2 \mathrm{H}), 6.20(\mathrm{dt}, \mathrm{J} 7,14 \mathrm{~Hz}, 1 \mathrm{H}), 1.8(\mathrm{~d}, \mathrm{~J} 7 \mathrm{~Hz}, 3 \mathrm{H}$ ) 
ppm. ${ }^{31} \mathrm{P}-\mathrm{NMR}\left\{\left(\mathrm{CD}_{3}\right) 2 \mathrm{CO} / 200 \mathrm{MHz}\right\} 20.83$ ppm. MS: m/e 268( $\left.{ }^{+}, 100\right), 253(83), 202(59)$, $183(64), 144(52), 115(11), 129(44), 77(40)$.

3. 2. 9. $\mathrm{IE}, 3 \mathrm{E}$-hexadienyldiphenyl phosphine oxide (5b)

White crystal, m. p. 119-121 oC. IR (KBr) $2900,1620,1560,1420,1160,1100,988 \mathrm{~cm}^{-1}$.

'H-NMR $\left\{\left(\mathrm{CD}_{3}\right)_{2} \mathrm{CO} / 200 \mathrm{MHz}\right\}: 7.75(\mathrm{~m}, 4 \mathrm{H}), 7.5(\mathrm{~s}, 6 \mathrm{H}), 7.10$ (ddd, J $10,16.5,18.5 \mathrm{~Hz}$, $1 \mathrm{H}), 6.50(\mathrm{~m}, 2 \mathrm{H}), 6.20(\mathrm{dt}, \mathrm{J} 7,13.5 \mathrm{~Hz}, 1 \mathrm{H}) 2.2(\mathrm{~m}, 2 \mathrm{H}), 1.0(\mathrm{t}, \mathrm{J} 7 \mathrm{~Hz}, 3 \mathrm{H}) \mathrm{ppm}$. ${ }^{31} \mathrm{P}-$ NMR $\left\{\left(\mathrm{CD}_{3}\right)_{2} \mathrm{CO} / 90 \mathrm{MH}\right\} \cdot 20.92 \mathrm{ppm}$. MS: m/e 282(M+, 64), 267(12), 253(100), 201 (63), $183(48), 158(26), 129(28), 77(19)$. Anal. Caled for $\mathrm{C}_{18} \mathrm{H}_{18} \mathrm{OP}, \mathrm{C}, 76.58 ; \mathrm{H}, 6.78 ;$ Found: C, 76. $50, \mathrm{H}, 6.47$.

3. 2. 10. 1E, 3E-heptadienyldiphenyl phosphine oxide ( $5 \mathrm{c}$ )

White crystal, m. p. $91-93^{\circ} \mathrm{C}$. IR (KBr) $2950,2850,1620,1560,1420,1100,985 \mathrm{~cm}^{-1}$. 'H-NMR $\left\{\left(\mathrm{CD}_{3}\right), \mathrm{CO} / 200 \mathrm{MHz}\right\}: 7.75(\mathrm{~m}, 4 \mathrm{H}), 7.5(\mathrm{~s}, 6 \mathrm{H}), 7.10(\mathrm{ddd}, \mathrm{J} 9.5,16.7,18.6$ $\mathrm{Hz}, 1 \mathrm{H}), 6.5(\mathrm{~m}, 2 \mathrm{H}), 6.19(\mathrm{dt}, \mathrm{J} 7,14.3 \mathrm{~Hz}, 1 \mathrm{H}), 2.08(\mathrm{~m}, 2 \mathrm{H}), 1.30(\mathrm{~m}, 2 \mathrm{H}), 0.95(\mathrm{t}, \mathrm{J}$ $7 \mathrm{~Hz}, 3 \mathrm{H}) \mathrm{ppm} . \quad{ }^{31} \mathrm{P}-\mathrm{NMR}\left\{\left(\mathrm{CD}_{3}\right)_{2} \mathrm{CO} / 90 \mathrm{MHz}\right\}: 21.07 \mathrm{ppm} . \mathrm{MS}: \mathrm{m} / \mathrm{e} 297\left(\mathrm{M}^{+}+1,100\right), 253$ (35), $201(31), 183(6), 115(5), 77(12)$. Anal. Calcd for $\mathrm{C}_{10} \mathrm{H}_{21} \mathrm{OP}$ : C, 77. 01; H, 7.14; Found; C. $76.80 ; \mathrm{H}, 6.86$.

3. 2. 11. 1E, 3E-octadienyldiphenyl phosphine oxide (5d)

White crystal, m. p. $73-75^{\circ} \mathrm{C}$. IR (KBr) $2952,2851,1620,1565,1420,1165,985 \mathrm{~cm}^{-1}$.

${ }^{1} \mathrm{H}-\mathrm{NMR}\left\{\left(\mathrm{CD}_{3}\right), \mathrm{CO} / 200 \mathrm{MHz}\right\}: 7.76(\mathrm{~m}, 4 \mathrm{H}), 7.52(\mathrm{~s}, 6 \mathrm{H}), 7.11$ (ddd, J 10, 16.5, 18.5 Hz, $1 \mathrm{H}), 6.48(\mathrm{~m}, 2 \mathrm{H}), 6.20(\mathrm{dt}, \mathrm{J} 7,14 \mathrm{~Hz}, 1 \mathrm{H}), 2.10(\mathrm{~m}, 2 \mathrm{H}), 1.30(\mathrm{~m}, 4 \mathrm{H}), 0.95(\mathrm{t}, \mathrm{J} 7 \mathrm{~Hz})$ ppm. ${ }^{31} \mathrm{P}-\mathrm{NMR}\left\{\left(\mathrm{CD}_{3}\right)_{2} \mathrm{CO} / 90 \mathrm{MHz}\right\}: 20.87 \mathrm{ppm}$. MS: m/e $311\left(\mathrm{M}^{+}+1,95\right), 281(100), 253$ (8), $231(96), 201(23), 177(7), 77$ (11). Anal. Caled for $\mathrm{C}_{20} \mathrm{H}_{23}$ OP: C, 77. 40; H, 7. 47; Found: $\mathrm{C}, 77.86 ; \mathrm{H}, 7.52$.

\section{Acknowledgements}

This work was finished at Shanghai Institute of Organic Chemistry, Academia Sinica. Financial support from the National Natural Science Foundation of China and Academia Sinica is gratefully acknowledged.

\section{References}

1 (a) B. M. Trost and T. Schmidt, J. Am. Chem. Soc., 110 (1988) 2301.

(b) D. Ma, Y. Lin, X. Lu and Y. Yu, Tetrahedron Lett. , 29 (1988) 1045.

(c) D. Ma, Y. Yu and X. Lu, J. Org. Chem., 54 (1989) 1105.

2 D. Ma and X. Lu, Tetrahedron Lett. , 30 (1989) 843.

3 D. Ma and X. Lu, Tetrahedron, 46 (1990) 3189.

4 (a) D. Ma and X. Lu, J. Chem. Soc., Chem. Commun. , (1989) 890.

(b) D. Ma and X. Lu, Tetrahedron Lett. , 30 (1989) 2109. 
(c) X. Lu, C. Guo and D. Ma, J. Org. Chem., 56 (1991) 6712.

5 Y. Wang, X. Jin and Y.Xu, Acta Chimica Sinica, 44 (1986) 235.

6 X. Lu, J. Ji, D. Ma and W. Shen, J. Org. Chem., 56 (1991) 5774.

7 B. N. Noknh and A. A. NempoB, Zh. Obsch. Khim., 34 (1964) 1174.

8 (a) E. N. Ofitserrov, A. A. Karelov and F. S. Bilalov, Zh. Obshch. Khim. 53 (1983) 511.

(b) J. C. Buck, F. Ellis and P. C. North, Tetrahedron Lett. , 23 (1982) 4161.

9 M. S. Chattha and A. M. Aguiar, J. Org. Chem. , 36 (1971) 2719.

10 Y.Xu, L. Jin, G. Huang and Y. Wang, Acta Chimica Sinica, 44 (1986) 183.

11 T. Minami, S. Tokumasu and I. Hirao, Bull. Chem. Soc. Jpn. , 58 (1985) 2139.

12 H. J. Bestmann, K. H. Koschatzky and A. Plenchette, Liebigs. Ann. Chem. , (1982) 536

13 U. C. Oxpuuehko and A. A. NempoB, Zh. Obshch. Khim., 38 (1968) 2124; 40 (1970) 804 .

Received: June 12, 1995 - Accepted: June 23, 1995 - Received in revised camera-ready format: July 13, 1995 
